\title{
Human Colorectal Cancer Stage-dependent Global DNA Hypomethylation of Cancer-associated Fibroblasts
}

\author{
EDUARD LING ${ }^{1,2}$, AMIT RINGEL ${ }^{2,3,4}$, INA SIGAL-BATIKOFF ${ }^{2,3,4}$, \\ NAIM ABU-FREHA ${ }^{2,4}$, HANANYA VAKNINE ${ }^{5}$, WLEDY FRIAH ${ }^{2,4}$, AVRAHAM RESHEF ${ }^{2,6}$, \\ ILIA PINSK ${ }^{2,7}$, ALEXANDER FICH $^{2,4}$ and SERGIO LAMPRECHT ${ }^{2,3,4}$ \\ ${ }^{1}$ Pediatrics Department B, Saban Pediatric Medical Center for Israel, \\ ${ }^{4}$ Institute of Gastroenterology and Hepatology, ${ }^{6}$ Surgery Department B, and \\ ${ }^{7}$ Unit of Colorectal Surgery, Soroka University Medical Center, Beer Sheva, Israel; \\ ${ }^{2}$ Faculty of Health Sciences, and ${ }^{3}$ Department of Clinical Biochemistry and Pharmacology, \\ Ben-Gurion University of the Negev, Beer Sheva, Israel; \\ ${ }^{5}$ Institute of Pathology, Wolfson Medical Center, Holon, Israel
}

\begin{abstract}
Background/Aim: Cancer-associated fibroblasts (CAFs) play an important role in tumor development and progression. The prevailing consensus favors the view that a specific epigenetic signature underpins the stable CAF phenotype. The aim of the present study was to assess global DNA methylation in CAFs during the adenoma-carcinoma sequence in non-familial sporadic human colorectal cancer (CRC). Patients and Methods: Immunohistochemical staining of nuclear 5-methylcytosine (5'-meCyt) was performed in matched samples of colonic tumor tissue and normal colonic mucosa excised from six patients with adenomas and four with adenocarcinomas. The staining intensity was expressed semiquantitatively as the immunohistochemical staining score (ISS). Results: ISS values of human colonic CAFs and adenomatous samples were $14.00 \pm 2.2$ and $14.08 \pm 1.8$, respectively, showing no statistically significant difference. In contrast, a marked trend was found towards global DNA hypomethylation in CAFs from adenocarcinomatous specimens compared to matched normal mucosa: ISS: $9.25 \pm 2.44$ (range $=6-11$ ) vs. $16.17 \pm 0.75$, respectively, $p<0.03$. Conclusion: Final stages of cancer development in CRC are associated with global DNA hypomethylation in stromal CAFs.
\end{abstract}

Traditionally, studies of neoplastic growth have focused on molecular events in tumor cells, whereas the stromal fibroblasts surrounding the tumor mass were considered

Correspondence to: Sergio Lamprecht, Ph.D., Department of Clinical Biochemistry and Pharmacology, Faculty of Health Sciences, Ben-Gurion University of the Negev, Beer Sheva, Israel. Tel: +972 8624606, Fax: +972 86479922, e-mail: sergiol@bgu.ac.il

Key Words: Methylation, fibroblasts, colorectal cancer. mere bystanders and, consequently, their role in cancer development and progression have remained largely neglected. Accruing information is now available showing that these mesenchymal cells are conscripted and educated by the adjacent cancer cells and as cancer-associated fibroblasts (CAFs) they participate in a malevolent alliance with their rogue neighbors, promoting their unrelenting growth and invasive program (1-4). Of note, however, evidence [reviewed in (5)] shows that this is not invariably the case: stromal components may act to impede early-stage tumorigenesis. In this scenario, the desmoplastic response represents an initial host defense designed to restrain the growth of the incipient tumor. A characteristic hallmark of CAFs is that they can be propagated in vitro and in vivo for extended periods of time without loss of their cancerpromoting phenotype $(6,7)$. Much evidence favors the prevailing opinion that this phenotypic stability is due to cancer-imposed epigenetic changes rather than to clonal somatic mutations (8-12) [reviewed in $(3,4,13-14)$ ].

Epigenetics refers to somatically inheritable changes in gene expression that do not require changes in DNA nucleotide sequence. A salient example of epigenetics is the control of gene activity by DNA methylation, i.e. the addition of a methyl group to the $5^{\prime}$ carbon of the pyrimidine cytosine within a DNA CpG dinucleotide sequence, an enzymatic reaction catalyzed by DNA methyltransferases (DNMTs) (Figure 1). Methylation extends through the promoter and start site of a gene and typically prohibits the critical interaction of transcription factors with DNA-response elements. Thereby, DNA site-specific hypermethylation is associated with heterochromatin and gene silencing. Conversely, DNA hypomethylation results in transcriptionally active euchromatin leading to brisk gene expression (15). 
Global DNA hypomethylation has been described in CAFs derived from a large number of solid tumors including human gastric carcinomas $(10,11)$. An early work reported global DNA hypomethylation in the stromal proteoglycan versican gene in human colonic carcinoma cells (16), an interesting observation since full-length human recombinant versican expression in cultured murine fibroblasts was shown to induce a myofibroblast-like phenotype (17), a wellrecognized hallmark of CAFs.

The aim of the present study was to assess global DNA methylation in CAFs at the various stages of the human nonfamilial sporadic CRC adenoma-adenocarcinoma sequence using a validated immunohistochemical assay that reports the staining intensity of 5'-methyl cytosine (5'-meCyt) in the cell nucleus.

\section{Materials and Methods}

Patients. Colonic tissues were obtained from biopsies taken from eight patients at various stages of the CRC adenoma-carcinoma sequence who underwent colonoscopy at the Institute of Gastroenterology and Hepatology, Soroka University Medical Center, Beer Sheba, Israel. This group consisted of two patients with adenocarcinomas and six with adenomas. Specimens of colonic adenocarcinomas were also obtained from two patients who underwent surgery at the Surgery Division, Soroka University Medical Center, Beer Sheba, Israel, and, collectively, the colonic adenocarcinoma group consisted of four patients, The adenoma group consisted of three patients with tubular adenomas (benign polyps) and three with tubulovillous adenomas (prone to malignancy) diagnosed by a senior pathologist.

Biopsy specimens of intact colonic mucosa were obtained from macroscopically normal colonic mucosa located at least $5 \mathrm{~cm}$ from the tumor margin. All tissue specimens were fixed in formalin and embedded in paraffin, and $4 \mu \mathrm{m}$ tissue sections were used for further studies. All patients signed an informed consent form. The study was approved by the Ethics Committee of Soroka University Medical Center in compliance with the ethical standards of the Helsinki Declaration: Clinical numbers: 0140-12-SOR, 033-14-SOR.

Immunohistochemistry. Figure 1 shows the epigenetic reaction leading to methylation of the DNA nucleotide cytosine. All chemicals of highest purity grade available were purchased from Sigma-Aldrich Corp. (St. Louis, MO, USA) unless stated otherwise. Nuclear 5'-meCyt was detected using a mouse monoclonal antibody (162 33 D3; Calbiochem, Merck Millipore, Darmstadt, Germany) raised against 5-meCyt conjugated to ovoalbumin, tested previously in human gastric CAFs (10) and in a human colonic myofibroblast cell line (Ling E, unpublished observation). The protocol was as described by Jiang et al. (10) with minor modifications. Briefly, tissue sections were deparaffinized in xylene and rehydrated in graded alcohol series. Antigen retrieval was performed in boiling $100 \mathrm{mM}$ citrate buffer ( $\mathrm{pH}$ 6.0) for $20 \mathrm{~min}$ with subsequent cooling at room temperature for an additional $20 \mathrm{~min}$. Following wash in phosphate-buffered saline (PBS), the tissue sections were exposed to $3.5 \mathrm{~N} \mathrm{HCl}$ for $15 \mathrm{~min}$ at room temperature to induce DNA denaturation. Following washing in PBS and 20-min incubation with $0.3 \% \mathrm{H}_{2} \mathrm{O}_{2}$ in absolute methanol for quenching endogenous

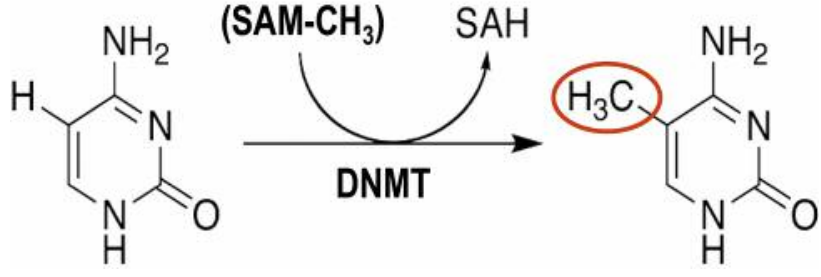

Figure 1. Methylation of DNA cytosine catalyzed by DNA methyltransferase (DNMT). A methyl group $\left(-\mathrm{CH}_{3}\right)$ is enzymatically transferred from $S$-adenosylmethionine (SAM) to the 5' carbon of the pyrimidine cytosine (in red) within a DNA CPG dinucleotide sequence. The monoclonal antibody used in this study specifically recognizes the methylated cytosine.

peroxidase activity, and an additional wash in PBS, tissue sections were incubated with $1.5 \%$ horse serum for $60 \mathrm{~min}$ at room temperature and then with anti-5'meCyt at 1:500 dilution in $1 \%$ albumin solution in PBS for 24 hours at $4^{\circ} \mathrm{C}$. Antibody detection was performed using Vectastain $\mathrm{ABC}$ kit (Vector Laboratories, Burlingame, CA, USA) with diaminobenzidine substrate according to the manufacturer's instructions. Slides were counterstained with hematoxylin, dehydrated and mounted. The majority of slides contained both pathological and normal tissue sections, when such slides were not available, both control and pathological tissue sections were treated identically.

Pathological evaluation. The intensity of nuclear staining of fibroblasts adjacent to adenomas or carcinomas was compared to that of resident fibroblasts in the lamina propria of normal colonic mucosa. Additionally, the intensity of staining of fibroblasts was compared to staining of normal and malignant epithelial cells and of lymphocytes found in the stromal tissue. These types of cells (epithelial cells and accompanying lymphocytes) were chosen to serve as internal control for two reasons: firstly, the nuclear staining of these cells for 5'-meCyt is well documented (18) and, secondly, the variability of staining of epithelial cells and lymphocytes was low. Nuclear staining intensity was scored as low (score 1), moderate (score 2) and intense (score 3), respectively. The high intensity score of 3 was defined as the staining intensity of cells in the adjacent epithelial cells and of lymphocytes. Cell scoring for nuclear 5'-meCyt staining was performed by a senior pathologist using a double-headed light microscope. The scoring system was based on global assessment of staining in all fibroblast nuclei in the entire high-power field compared with that in epithelial cells in the same field. The intensity of staining was evaluated in six randomized high-power fields of $2 \mathrm{~mm}^{2}$ per slide and scoring was expressed as the average density per $1 \mathrm{~mm}^{2}$ field.

Statistical analysis. Wilcoxon signed-rank test was used to evaluate the association between dependent variables. Each slide was assigned a final score which was based on the sum of individual intensity scores of each power field. Four groups were defined for statistical purposes: adenomatous specimens versus normal tissue specimens, and adenocarcinomatous samples versus normal tissue samples. The nuclear staining intensity scores of tumor surgical specimens were pooled with those of biopsy specimens. The statistical analysis was performed using IBM SPSS software (SPSS 

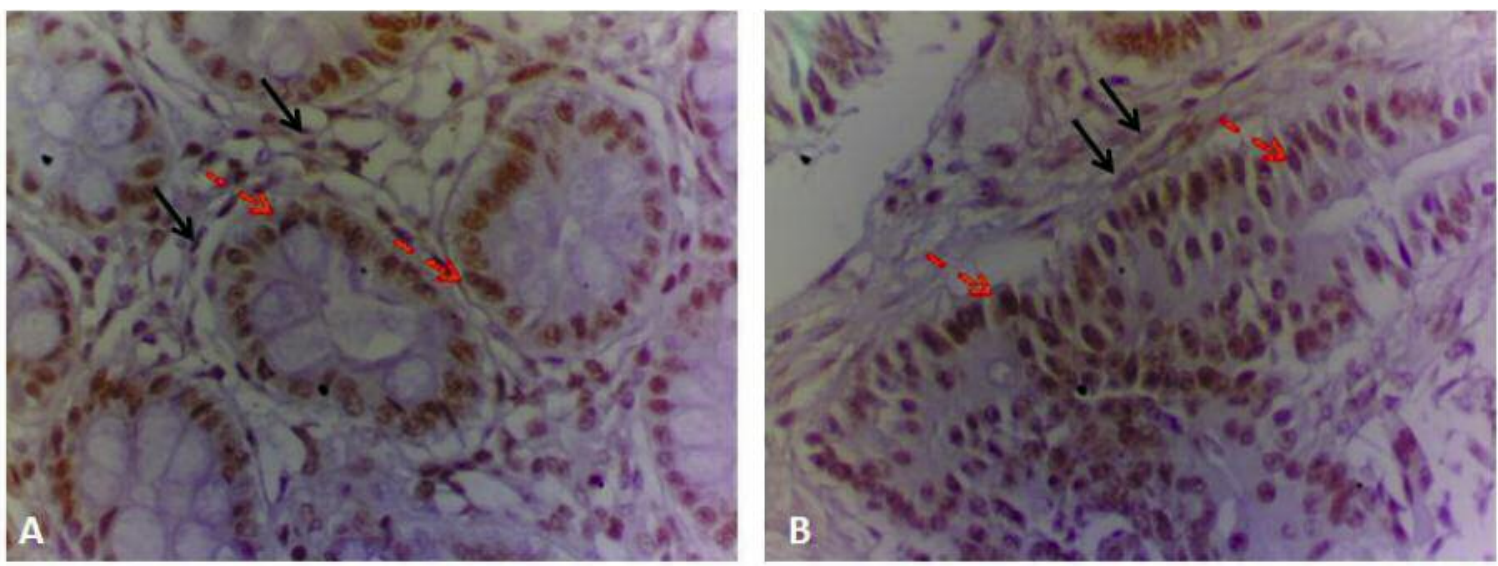

Figure 2. Immunostaining for nuclear 5'-methyl cytosine (5'-meCyt) of normal human stromal colonic fibroblasts (A) and cancer-associated fibroblasts $(C A F s)(B)$. A: The intensity of 5'-meCyt staining in normal stromal fibroblasts (black arrows) is markedly higher than that expressed in CAFs (B), and comparable to that of normal colonic epithelial cells (red dashed arrows). B: The intensity of 5'-meCyt staining in CAFs (black arrows), markedly lower compared to normal stromal fibroblasts (A) and to adjacent adenocarcinomatous epithelial cells (red dashed arrows), indicates global DNA hypomethylation. Representative slides (original magnification $\times 400$ ) are shown.

19.0 for Windows; SPSS Inc, Chicago, IL, USA); a value of $p<0.05$ was considered statistically significant.

\section{Results}

Global DNA methylation in stromal fibroblasts of colonic tubular and tubulovillous adenomas. Tissue specimens of six patients with colonic adenomas were examined. In all samples, the nuclei of epithelial cells (normal, adenomatous and carcinomatous), stromal fibroblasts and lymphocytes within the lamina propria stained positively for 5-meCyt (data not shown). The mean nuclear intensity score of 5'-meC staining of fibroblasts in normal colonic mucosa was $14.08 \pm 1.83$ (range=12-16), whereas the respective value for CAFs in the stroma of colonic adenomas was found to be $14.00 \pm 2.2$ (range $=11-17$ ): This difference was not statistically significant.

Global DNA hypomethylation in stromal fibroblasts of colonic adenocarcinomas. Colonic mucosa specimen of four patients with colonic adenocarcinomas were examined. In all samples, nuclei of epithelial cells, stromal fibroblasts and intraepithelial lymphocytes stained positively for 5'-meC; however, a markedly reduced nuclear staining intensity of stromal CAF adenocarcinoma was consistently found compared to their counterparts in normal mucosa. The score of nuclear staining intensity revealed a significant $(p<0.03)$ loss of nuclear staining of colonic adenocarcinoma CAFs compared to stromal fibroblasts surrounding the normal colonic mucosa (Figure 2): The nuclear staining intensity score in CAFs was 9.25 \pm 2.44 (range=6-13) vs. 16.17 \pm 0.75 (range $=15-17$ ) in normal stromal fibroblasts.
No difference in staining intensity of epithelial cells and intraepithelial lymphocytes between normal tissue and adenocarcinoma tissue was found (Figure 2).

\section{Discussion}

Our results indicate that, similarly to other gastrointestinal regions (10), significant changes in the epigenetic signature are a hallmark of colonic CAFs in human CRC. Notably, global DNA hypomethylation, assessed by immunostaining of nuclear 5'-meCyt, was fully established in stromal colonic $\mathrm{CAFs}$ at the adenocarcinoma CRC stage. Interestingly, no difference in nuclear 5'meCyt intensity staining was noted between colonic benign polyps or polyps prone to malignancy and normal stromal fibroblasts.

In a series of preliminary experiments (19) designed to provide direct evidence that cancer cells instruct colonic fibroblasts to acquire a CAF-like phenotype, we previously assessed global DNA methylation in a human normal colonic myofibroblast line (CCD-18Co) co-cultered in a Transwell system with HT-29 cells, a human colonic adenocarcinoma cell line. Global DNA methylation was quantified by a microplate-based assay. The exposure of colonic fibroblasts to cancer cells resulted in a marked time-dependent loss of DNA methylation. These findings, albeit preliminary, indicate that sustained paracrine cross-talk of CCD-18Co cells with cancer cells changes the epigenetics of colonic fibroblasts.

Our findings concord with those reported by Mrazek et al. (20). These investigators used genomic-wide genemicroarray expression and Illumina-based methylation analysis to study cultured colonic CAFs collected from 
stroma surrounding colonic adenocarcinomas. Their results indicated differential expression of genes and DNA methylation of promoter regions specific to CAFs. As noted in other solid tumor types (11), global DNA hypomethylation was associated with focal DNA hypermethylation.

What are the putative molecular mechanisms underpinning changes in the epigenetic landscape of colonic CAFs in terms of global DNA methylation? Neoplastic cells induce a chronic inflammatory response that builds up an inflammatory pro-tumorigenic microenvironment (TME) $(21,22)$. In addition, CAFs maintain the inflammatory TME by expressing a pro-inflammatory gene signature and by acting as brisk recruiters of pro-inflammatory cells. Therefore, in a vicious self-sustaining train of events, both tumor cells and CAFs contribute to the TME inflammasome (23-25).

A vast body of evidence is available showing that proinflammatory cytokines are able to exert their action on key components of the epigenetic machinery $(12,26,27)$. Among them, transforming growth factor- $\beta$ (TGF $\beta$ ) plays a determinant role and findings indicate that a frequent site of TGF $\beta$ intervention is the regulation of DNMTs involved in DNA methylation [Figure 1 (28), reviewed in (29). Interestingly, Hawinkels and co-workers presented evidence that levels of active TGF $\beta 1$ are increased in human colonic tissue at the adenocarcinoma stage (30), to wit, at the CRC stage in which frank changes in $\mathrm{CAF}$ epigenetic landscape were observed in this study. Of note, a high level of TGF $\beta$ in CRC tumors correlates with disease relapse (31).

As discussed previously, DNA hypomethylation is associated with proficient chromatin and brisk activity of transcription factors. We surmise that among the newlyexpressed genes are well-recognized markers of the CAF trait such as cyclooxygenase (COX2), a potential oncogenic enzyme, and fibroblastic-activation protein $(F A P)$, an enzyme involved in the remodeling of the extracellular matrix (4, 24). Concordant with this view, FAP was shown to be expressed in CAFs surrounding colorectal carcinomas (32), and high FAP expression in CRC was shown to be associated with poor patient prognosis (33).

Epigenetic changes, unlike genetic ones, are potentially reversible and can be interfered with by drugs (14). Our observation that colonic CAFs express frank changes in their epigenetic signature predominantly at the adenocarcinoma stage of CRC may indicate a critical timing when administration of drugs able to reverse epigenetic changes in CRC could be beneficial.

A limitation of the present study is that only global DNA methylation in CRC CAFs was examined, and a deliberate characterization of the DNA methylome must include the assessment of gene promoter site-specific methylation signature. Moreover, our approach precludes any information on possible phenotypic and functional heterogeneity of colonic stromal CAFs (34). An attractive line of research, poorly explored to date, should also address specific histone post-translational modifications in stromal fibroblasts. We surmise that in addition to changes in the DNA methylome, histone changes greatly contribute to the stable CAF phenotype and, thereby, they sustain the role of CAFs in promoting the opportunistic growth agenda of cancer cells.

\section{Conflicts of Interests}

None.

\section{References}

1 Kalluri R and Zeisberg M: Fibroblasts in cancer. Nat Rev Cancer 6: 392-401, 2006.

2 Orimo A and Weinberg R: Stromal fibroblasts in cancer: A novel tumor-promoting cell type. Cell Cycle 5: 1597-1601, 2006.

3 Madar S, Goldstein I and Rotter V: Cancer-associated fibroblasts - more than meets the eye. Trends Mol Med 19: 447-453, 2013.

4 Polanska U and Orimo A: Carcinoma-associated fibroblasts: non-neoplastic tumour-promoting mesenchymal cells. J Cell Physiol 228: 1651-1657, 2013.

5 Delinasios JG, Angeli F, Koumakis G, Kumar S, Kang WH, Sica G, Iacopino F, Lama G, Lamprecht S, Sigal-Batikoff I, Tsangaris GT, Farfarelos CD, Farfarelos MC, Vairaktaris E, Vassiliou S and Delinasios GJ: Proliferating fibroblasts and HeLa cells cocultured in vitro reciprocally influence growth patterns, protein expression, chromatin features and cell survival. Anticancer Res 35: 1881-1916, 2015.

6 Orimo A, Gupta PB, Sgroi DC, Arenzana-Seisdedos F, Delaunay T, Naeem R, Carey VJ, Richardson AL and Weinberg RA: Stromal fibroblasts present in invasive human breast carcinomas promote tumor growth and angiogenesis through elevated SDF1/CXCL12 secretion. Cell 121: 335-348, 2005.

7 Olumi AF, Grossfeld GD, Hayward SW, Carroll PR, Tlsty TD and Cunha GR: Carcinoma-associated fibroblasts direct tumor progression of initiated human prostatic epithelium. Cancer Res 59: 5002-5011, 1999.

8 Fiegl H, Millinger S, Goebel G, Müller-Holzner E, Marth C, Laird PW and Widschwendter M: Breast cancer DNA methylation profiles in cancer cells and tumor stroma: association with $H E R-2 / n e u$ status in primary breast cancer. Cancer Res 66: 29-33, 2006.

9 Hu M, Yao J, Cai L, Bachman KE, van den Brûle F, Velculescu $\mathrm{V}$ and Polyak K: Distinct epigenetic changes in the stromal cells of breast cancers. Nature Genetics 37: 899-905, 2005.

10 Jiang L, Gonda TA, Gamble MV, Salas M, Seshan V, Tu S, Twaddell WS, Hegyi P, Lazar G, Steele I, Varro A, Wang TC and Tycko B: Global hypomethylation of genomic DNA in cancer-associated myofibroblasts. Cancer Res 68: 9900-9908, 2008.

11 Vizoso M, Puig M, Carmona FJ, Maqueda M, Velásquez A, Gómez A, Labernadie A, Lugo R, Gabasa M, Rigat-Brugarolas LG, Trepat X, Ramírez J, Moran S, Vidal E, Reguart N, Perera A, Esteller ML and Alcaraz J: Aberrant DNA methylation in non-small cell lung cancer-associated fibroblasts. Carcinogenesis 36: 1453-1463, 2015. 
12 Albrengues J, Bertero T, Grasset E, Bonan S, Maiel M, Bourget I1, Philippe C, Herraiz Serrano C, Benamar S, Croce O,SanzMoreno V, Meneguzzi G, Feral CC, Cristofari G and Gaggioli $\mathrm{C}$ : Epigeneitic switch drives the conversion of fibroblasts into proinvasive cancer-associated fibroblasts. Nature Comm 6: 10204, 2015.

13 Rudnick S and Kuperwasser C: Stromal biomarkers in breast cancer development and progression. Clin Exp Metastasis 29: 663-72, 2012.

14 Gonda T, Varro A, Wang T and Tycko B: Molecular biology of cancer associated fibroblasts: Can these cells be targeted in anticancer therapy? Semin Cell Dev Biol 21: 2-10, 2010.

15 Kouzarides T: Chromatin modifications and their function. Cell 128: 693-705, 2007.

16 Adany $\mathrm{R}$ and Iozzo $\mathrm{R}$ : Altered methylation of versican proteoglycan gene in human colon carcinoma. Biochem Biophys Res Comm 171: 1402-1413, 1990.

17 Carthy JM, Meredith AJ, Boroomand S, Abraham T, Luo Z, Knight D and McManus BM: Versican V1 overexpression induces a myofibroblast-like phenotype in cultured fibroblasts PLoS One 10: e0133056, 2015.

18 Hinoi T, Akyol A, Theisen BK, Ferguson DO, Greenson JK, Williams BO, Cho KR and Fearon ER: Mouse model of colonic adenoma-carcinoma progression based on somatic Apc inactivation. Cancer Res 67: 9721-9730, 2007.

19 Lamprecht S, Deliniasos J, Fich A and Abu-Freha N: Do cancer cells impose epigenetic changes on normal fibroblasts? A colonoriented preliminary inquiry. Anticancer Res 34: 6020-6021, 2014.

20 Mrazek AA, Carmical JR, Wood TG, Hellmich MR, Eltorky M, Bohanon FJ and Chao C: Colorectal cancer-associated fibroblasts are genotypically distinct. Curr Cancer Ther Rev 10: 97-218, 2014.

21 Rokavec M, Öner M and Hermeking H: lnflammation-induced epigenetic switches in cancer. Cell Mol Life Sci 73: 23-39, 2016.

22 Abu-Remaileh M, Bender S, Raddatz G, Ansari I, Cohen D, Gutekunst J, Musch T, Linhart H, Breiling A, Pikarsky E,Bergman Y and Lyko F: Chronic inflammation induces a novel epigenetic program that is conserved in intestinal adenomas and in colorectal cancer. Cancer Res 75: 2120-2130, 2015.

23 Erez N, Glanz S, Raz Y, C, Avivi C and Barshack I: Cancer associated fibroblasts express pro-inflammatory factors in human breast and ovarian tumors. Biochem Biophys Res Commun 437: 397-402, 2013.

24 Erez N, Truitt M, Olson P, Arron S and Hanahan D: Cancerassociated fibroblasts are activated in incipient neoplasia to orchestrate tumor-promoting inflammation in an NF-kappaBdependent manner. Cancer Cell 17: 135-147, 2010.
25 Servais C and Erez N: From sentinel cells to inflammatory culprits: cancer-associated fibroblasts in tumour-related inflammation. J Pathol 229: 198-207, 2013.

26 Landskron G, de la Fuente M, Thuwajit P, Thuwajit C and Hermoso MA: Chronic inflammation and cytokines in the tumor microenvironment. J Immunol Res 2014: 149185, 2014.

27 Hodge DR, Peng B, Cherry JC, Hurt EM, Fox SD, Kelley JA, Munroe DJ and Farrar WL: Interleukin 6 supports the maintenance of p53 tumor-suppressor gene promoter methylation. Cancer Res 65: 4673-4682, 2005.

28 Pan X, Chen Z, Huang R, Yao Y and Ma G: Transforming growth factor $\beta 1$ induces the expression of collagen type I by DNA methylation in cardiac fibroblasts. Plos One 8: e60335, 2013.

29 Pickup M, Novitskiy S and Moses H: The roles of TGF $\beta$ in the tumour microenvironment. Nat Rev Cancer 13: 788-799, 2013.

30 Hawinkels LJ, Verspaget HW, van der Reijden JJ, van der Zon JM, Verheijen JH, Hommes DW, Lamers CB and Sier CF: Active TGF-beta1 correlates with myofibroblasts and malignancy in the colorectal adenoma-carcinoma sequence. Cancer Sci 100: 663-670, 2009.

31 Calon A, Lonardo E, Berenguer-Llergo A, Espinet E, HernandoMomblona X, Iglesias M, Sevillano M, Palomo-Ponce S, Tauriello DVF, Byrom D, Cortina C, Morral C, Barceló C, Tosi S, Riera A, Attolini CS-O, Rossell D, Sancho E and Batlle E: Stromal gene expression defines poor-prognosis subtypes in colorectal cancer. Nat Genet 47: 320-329, 2015.

32 Koczorowska MM, Tholen S, Bucher F, Lutz L, Kizhakkedathu JN, De Wever O, Wellner UF, Biniossek ML, Stahl A, Lassmann $S$ and Schilling O: Fibroblast activation protein- $\alpha$, a stromal cell surface protease, shapes key features of cancer associated fibroblasts through proteome and degradome alterations. Mol Oncol 10: 40-58, 2016.

33 Wikberg M, Edin S, Lundberg I and van Guelpen B: High intratumoral expression of fibroblast activation protein (FAP) in colon cancer is associated with poorer patient prognosis. Tumour Biol 34: 1013-1020, 2013.

34 Ohlund D, Elyada E and Tuveson D. Fibroblast heterogeneity in the cancer wound: J Exp Med 211: 1503-1523, 2014.
Received June 21, 2016

Revised August 11, 2016

Accepted August 17, 2016 